\title{
A piezoelectric immunosensor for Leishmania chagasi antibodies in canine serum
}

\author{
Joilson Ramos-Jesus • Kellyanne A. Carvalho • Rosana A. S. Fonseca • \\ Geraldo G. S. Oliveira • Stella M. Barrouin Melo • Neuza M. Alcântara-Neves • \\ Rosa F. Dutra
}

Received: 26 December 2010/Revised: 27 April 2011 /Accepted: 20 May 2011 /Published online: 14 June 2011

(C) Springer-Verlag 2011

\begin{abstract}
The American visceral leishmaniasis is an important cause of morbidity and mortality in Brazil for both humans and dogs. Attempts to make a diagnosis of this disease need to be improved, especially in endemic areas, and in the tracking and screening of asymptomatic dogs, which are their main host in urban areas. A quartz crystal microbalance immunosensor for the diagnosis of the canine visceral leishmaniasis using a recombinant antigen of Leishmania chagasi (rLci2B-NH6) was developed. The rLci2B-NH6 was tightly immobilized on a quartz crystal gold electrode by self-assembled monolayer based on short-chain length thiol. The strategy was the use of the antigen-histidine tail covalently linked to glutaraldehyde performing a Schift base which permits a major exposure of epitopes and a reduced steric hindrance. The
\end{abstract}

J. Ramos-Jesus $\cdot$ R. A. S. Fonseca • R. F. Dutra $(\bowtie)$

Laboratório de Engenharia Biomédica,

Universidade Federal de Pernambuco,

Av. Prof. Moraes Rego, 1235-Cidade Universitária,

Recife, Pernambuco 50670-901, Brazil

e-mail: rosa.dutra@pq.cnpq.br

R. F. Dutra

e-mail: rfiremandutra@yahoo.com.br

J. Ramos-Jesus $\cdot$ K. A. Carvalho $\cdot$ N. M. Alcântara-Neves

Departamento de Biointeração, Universidade Federal da Bahia,

Salvador, Bahia 40110-060, Brazil

G. G. S. Oliveira

Centro de Pesquisa Gonçalo Muniz,

Fundação Oswaldo Cruz, Bahia 40296-710, Brazil

S. M. B. Melo

Departamento de Patologia e Clínicas,

Universidade Federal da Bahia,

Salvador, Bahia 40110-060, Brazil immunosensor showed good results regarding sensitivity and reproducibility, being able to distinguish positive and negative canine serum for $L$. chagasi. Furthermore, the immunosensor can be reused through exposure to sodium dodecyl sulfate solution, which promotes the dissociation of antigen-antibody binding, restoring the sensor surface with immobilized biologically active antigens for further analysis.

Keywords Leishmania chagasi . Immunosensor.

Self-assembled monolayer. Piezoelectric

\section{Introduction}

American visceral leishmaniasis is a severe systemic disease caused by the intracellular protozoan, Leishmania chagasi, which occurs in Central and South America and some endemic areas of North America [1]. Dogs are considered the main host of the parasite, which is transmitted by the Lutzomyia longipalpis sandfly. The monitoring of canine visceral leishmaniasis (CVL) is important to control the parasite transmission to man [2]. Dogs usually develop the visceral form of the infection, with highly variable clinical appearances. The control of the CVL is difficult due to four main reasons: (1) The development of clinical symptoms from the moment of infection is unpredictable, being the signs common to other diseases [3]. (2) The splenic biopsy and bone marrow punctures necessary for parasitological diagnosis are invasive and need hospital facilities, the laboratory techniques are time consuming and variable in terms of sensitivity, which depends on the parasite load in the dog [4]. (3) Serological tests such as indirect immunofluorescence antibody test (IFAT) and enzyme linked immuno- 
sorbent assay (ELISA) for the antibodies detection have been widely used $[5,6]$. The sensitivity and specificity are $100 \%$ and $96.6 \%$ for ELISA, respectively, $100 \%$ and $65.5 \%$ for IFAT (cutoff at a 1:40 dilution), $100 \%$ and $83.4 \%$ for IFAT (cutoff at a 1:80 dilution), and $22.2 \%$ and $97.0 \%$ for eluate IFAT [7]. The ELISA is the best tool for the diagnosis of CVL among the serological techniques tested. However, the ELISA is time consuming because it relies on several biochemical steps. (4) The polymerase chain reaction (PCR) is highly sensitive and specific but is not practical for screening testing because it is time consuming and requires specialized skills and high cost [8]. Thus, the development of practical and real-time methods to detect quantitatively the reaction antigenantibodies in real time without labels or reagents is desirable.

Immunosensors are affinity ligand-based biosensing devices that couple immunochemical reactions to appropriate transducers. As a sensitive surface mass sensor, quartz crystal microbalance (QCM) has been extensively applied as transducer in biosensing for the detection of various pathogens [9]. The QCM is a variant of acoustic wave microsensors that are capable of ultrasensitive mass measurements. Under favorable conditions, a typical QCM can measure a mass change of $0.1-1 \mathrm{ng} / \mathrm{cm}^{2}$. QCM oscillates in a mechanically resonant shear mode under the influence of a high-frequency AC electric field which is applied across the thickness of the crystal. A piezoelectric sensor comprises a quartz crystal electrode consisting of two metal films (gold) deposited on both sides of the crystal coupling to an oscillator circuit. The mass change is measured when a biological adsorbent material interacts with its complementary species on the electrode surface, increasing the dielectric strength of the crystal and consequently reducing the oscillation of electric frequency [10]. The relationship between the frequency shift $(\Delta f)$ of the quartz crystal resonator and changes of its mass per unit surface area $(\Delta m)$, can be described by the Sauerbrey equation (Eq. 1):

$$
\Delta f=-\frac{2 f_{0}^{2}}{A\left(\mu_{\mathrm{q}} \rho_{\mathrm{q}}\right)^{1 / 2}} \Delta m
$$

where, $\mu_{\mathrm{q}}$ and $\rho_{\mathrm{q}}$ are the shear modulus and the density of the quartz (2.95 $10^{11} \mathrm{dyn} / \mathrm{cm}^{2}$ and $2.65 \mathrm{~g} / \mathrm{cm}^{3}$, respectively), $f_{0}$ is the fundamental oscillation frequency of the dry crystal, and $\Delta m$ is the mass of the material adsorbed on the surface per unit/area. As molecules adsorb onto the electrode, the oscillation frequency of the quartz crystal decreases and vice versa. The main advantage of piezoelectrical immunosensors is the possibility to directly monitor the immunoreactions in real time without the need to use labels or reagents; they can be portables for measurements in situ, and also it can be reusable, ready for another test, after dissociation of the immunocomplex, reducing the costs of analysis [11].

QCM and the combination of QCM with electrochemistry have been widely employed for the determination of metals deposited onto the crystal, studies of ion-transport processes in polymer films, biosensor developments, and investigations of the kinetics of adsorption/desorption of adsorbate molecules [12, 13]. Since piezoelectric immunosensor carries out direct measurements without labeled reagent, biomolecular receptor with high specificity to avoid significant interference of cross-reactions are required. Recently, the use of recombinant antigen based on DNA technology has provided serological diagnostics that are more reliable since the specificity of the antigen can be achieved easier than using whole extracts [14].

The way the receptors are fixed on the electrode surface and maintained during the reactions is strongly dependent on the specificity and sensitivity of the immunosensor. A great variety of methods suitable for the immobilization of immunoreceptors has been described in literature. Physical adsorption proves the easiest immobilization method, but it often suffers from random orientation and denaturation of immobilized biomolecules, yielding poor reproducibility [15]. A more stable immobilizations of receptors are obtained by covalent attachment yielding better reproducibility. Covalent immobilization of antigens or antibodies, however, can cause steric hindrance, which results in the loss of binding capability, which consequently reduces the sensitivity of QCM immunosensor [16]. Due to these reasons, the choice of a proper immobilization method is strongly required for the success of the immunosensors [17]. Selfassembled monolayers (SAMs) on gold supports are commonly performed by the ordering of organic thiols disposed in multiple layers resulting in high stability on the receptors immobilization. Moreover, one of the advantages of SAM includes their ease of preparation and the possibility of introducing different chemical functionalities resulting in an oriented immobilization [18]. The incorporation of the appropriate chemical functionality with some molecular level control into the highly ordered monolayers allows the preparation of surfaces with tailor-made properties. In this work, a reliable sensor was developed using the rLci2B-NH6 recombinant antigen of $L$. chagasi immobilized via SAM. The good sensitivity and specificity was achieved by the use of glutaraldehyde as a bi-functional agent to covalently link to the amine group of the histidine tail of the rLci3B leaving the antigenic portion free to antibodies bound without steric hindrance. 


\section{Experimental}

\section{Reagents}

Cysteamine (hydrochloride) glutaraldehyde, glycine and sodium dodecyl sulfate (SDS) were supplied by SigmaAldrich (USA) and potassium hexacyanoferrate(III) which was acquired from VETEC Inc. (Brazil). All other reagents were of analytical grade. The water used to prepare of all solutions was obtained from a Milli-Q water purification system (Millipore, USA).

\section{Recombinant antigen rLci2B-NH6}

The $L$. chagasi recombinant antigen (rLci2B-NH6) used was produced and purified by our group. The recombinant antigen was performed by a recombinant protein with $70 \mathrm{kDa}$ (rLci2B-NH6) bound to a group of six histidine fuzioned by their amine terminal group. The rLci2B-NH6 developed was homologue to the kinesin, a protein of the cytoskeleton of the Leishmania, located in the cynetoblast of the parasite and represented a fragment of the heat shock protein of $70 \mathrm{kDa}$ (HSP70) from $L$. chagasi. Recombinant DNA-derived $L$. chagasi antigen rLci2B-NH6 was obtained from the strain of Escherichia coli BL21(DE) pLysS containing the gene of $L$. chagasi rLci2B-NH6. The strain was cultivated and expanded in $0.1 \mathrm{mM}$ IPTG (isopropyl beta-D-thiogalactopyranoside) induction for the production and purification of recombinant antigen of $L$. chagasi (rLci2B-NH6) as a histidine-tagged protein. This antigen potential for serodiagnosis of CVL was previously tested by ELISA using sera from dogs with the following conditions: (a) 46 dogs naturally infected with Leishmania, detected by splenic aspirate and culture of CVL from endemic areas which were either polysymptomatic (21 dogs), oligosymptomatic (21 dogs), or asymptomatic (four dogs); (b) 31 dogs from Leishmania-free areas: seven with demodicosis, four with babesiosis, and 20 dogs with ehrlichiosis. All diseases were parasitologically confirmed. These assays showed sensitivities and specificities above 90\% (nonpublished data). L. chagasi kinesin was already tested with an immunoassay kit which showed nonreactive to the other trypanosomatidae [19, 20].

\section{Canine serum}

The canine serum samples were obtained from dogs naturally infected with $L$. chagasi from endemic area, detected by splenic aspirate cultivation. All infections were parasitologically confirmed. In addition, sera from healthy mongrel dogs housed in a nonendemic area were used as controls. The protocol for animal handling was approved by the Ethics Committee on Animal Use of the Research Center Gonçalo Muniz-Fiocruz-Bahia, Brazil.
Apparatus

The experimental setup consisted of Quartz Crystal Microbalance Research System (RQCM; Maxtec Inc., Salt Lake City, UT) with a high-performance phase-lock oscillator circuit that provides superior measurement stability over a high-frequency range. The RQCM was coupled to microcomputer and a potentiostat-galvanostat (Autolab, PGstat 12) which was, in turn, controlled by a GPES software (version 4.9).

All measurements of frequency and current were carried out using a tri-electrode system. The quartz crystal electrode $9 \mathrm{MHz}$ (Maxtec Inc.) as a working electrode, $\mathrm{Ag} / \mathrm{AgCl}$ as reference electrode and helicoidal platinum wire, as auxiliary electrode set in an electrochemical cell $(40 \mathrm{~mL})$. The quartz crystal electrode was an AT-cut type (diameter, $25.1 \mathrm{~mm}$ ) with $\mathrm{Au}$ electrodes on both sides (12.25 mm; $6.25 \mathrm{~mm}$ in diameter; $1.17 \mathrm{~cm}^{2}$ area).

\section{Immobilization of the rLci2B-NH6}

Prior the rLci2B-NH6 immobilization, the gold quartz crystal electrode was pretreated using $0.5 \mathrm{M} \mathrm{NaOH}$ solution for $3 \mathrm{~min}$, and washed $(3 \times)$ with ethanol and distilled water to obtain a clean gold surface. After that, a phosphatebuffered saline (PBS) solution, pH 7.4 containing $50 \mathrm{mM}$ cysteamine was applied to one side of the gold electrode for $2 \mathrm{~h}$, at room temperature to form an assembled monolayer. Then, the gold electrode was washed in PBS and incubated with $2.5 \%(v / v)$ glutaraldehyde solution for $45 \mathrm{~min}$ and washed with PBS three times. The modified gold electrode was incubated with rLci2B-NH6 antigen $(3 \mu \mathrm{g} / \mathrm{mL})$ for $1 \mathrm{~h}$. After the immobilization of the recombinant antigen, a solution of $50 \mathrm{mM}$ glycine was applied on the quartz crystal electrode to block the remaining reactive free aldehyde groups of the glutaraldehyde, in order to minimize the nonspecific binding. When not in use, the quartz crystal electrode was stored at 4 to $8{ }^{\circ} \mathrm{C}$.

Immunosensor reactivity to $L$. chagasi antibodies

Analytical response of the immunosensor to $L$. chagasi antibodies was evaluated by applying $200 \mu \mathrm{L}$ of canine serum samples diluted at 1:400 in PBS for $15 \mathrm{~min}$ on the active area of the quartz crystal electrode $(0.8 \mathrm{~cm}$ i.d.). The protocol for measurement of frequencies of the $r L c i 2 B$ NH6 immobilized quartz crystal electrode (with and without the tested serum) consisted of four PBS washings for $2 \mathrm{~min}$ each followed by its immersion in the electrochemical cell filled with PBS. The frequency shifts were proportional to the amount of antibodies against $L$. chagasi bound on the crystal electrode, as expected for a mass sensitive device. 
The dissociation of the immunocomplex to reuse the immobilized antigen was carried out by applying $0.1 \%(v / v)$ SDS for 3 min followed by three PBS washes.

\section{Statistical analysis}

The cutoff of the immunosensor was defined as the average delta frequency $(\mathrm{Hz})$ plus two standard deviations of values obtained by using sera from healthy controls dogs $(95 \%$ CI). The proportion of positives and negatives sera from $L$. chagasi-infected and non infected dogs (confirmed by splenic aspirate culture) allowed us to calculate the assay sensitivity and specificity, respectively. The statistical analysis was processed in the Origin 8 statistic program (OriginLab Co., USA). The results of the ELISA and immunosensor were compared by paired $t$ test, and the reproducibility of immunosensor was evaluated by variation within and between assays.

\section{Results and discussion}

\section{Immobilization of recombinant antigen}

A convenient way of tailoring the surface in the molecular level is through the inclusion of some well-known receptor molecules in the monolayers to be used as a support for molecular recognition at the surface through the formation of inclusion complexes by host-guest interaction. An amine group was introduced by aminoethanothiol (cysteamine) that was adsorbed on the quartz electrode by thiol-gold interaction. The amine groups of cysteamine provided reaction sites for covalently bind to the glutaraldehyde. The rLci2B-NH6 antigen was then immobilized through Schiff base via glutaraldehyde by histidine tail (Fig. 1a). Then, the immunosensor response was obtained by recognition of immobilized rLci2B-NH6 antigen.

The SAM formation is essentially an organization of molecules at the solid-liquid interfaces induced by strong chemisorption between the substrate and the head group. The main objective is to attain the capability of assembling individual molecules into highly ordered was assembled for obtaining of an ultrathin organic film of controlled thickness and desired functions. The monolayers can be characterized by a variety of methods including physical measurements like contact angle and wettability, different electrochemical investigations, Infrared and X-ray photoelectron spectroscopic methods, scanning probe microscopes etc. More specifically, electrochemical techniques are especially useful for monitoring monolayer quality for immunosensors [21]. For example, although a monolayer can completely cover the metal surface, some "pinhole," which are the result of imperfect adsorption of the

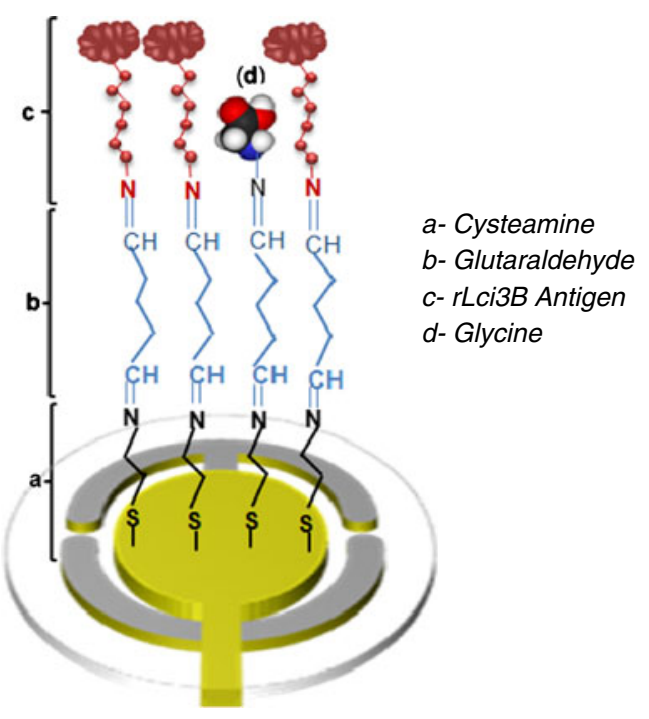

Fig. 1 Illustrative scheme of the rLci3B-NH6 immobilization on the quartz crystal electrode: (a) cysteamine, (b) glutaraldehyde, (c) antigen rLci3B-NH6, and (d) glycine

alkanethiol to the gold surface during the self-assembly step and/or subsequent loss of the thiol during rinsing, storage, or use, are always present and this type of pinhole causes a direct contact of redox active molecule with the electrode surface. Quantitative analysis of coverage can be calculated by cyclic voltammetric investigations [22]. Considerable attention has been focused on self-assembled monolayers of cysteamine on gold electrodes [23, 24]. Compared with the response on a bare gold electrode, $\mathrm{Fe}$ $(\mathrm{CN})_{6}{ }^{3-/ 4-}$ exhibits better reversibility on a cysteamine modified gold electrode due to the attractive electrostatic forces between the protonated amine groups of cysteamine and $\mathrm{Fe}(\mathrm{CN})_{6}{ }^{3-/ 4-}[25]$. The differences in the structures of carbon chains of monomers may lead to distinctions in the packing of the SAM formed by these thiols. The location and intensity (area) of the redox peaks can be used in the characterization of the monolayers with respect to their degree of coverage and stability. Figure 2 shows cyclic voltammograms using $1 \mathrm{mM} \mathrm{K}_{3} \mathrm{Fe}(\mathrm{CN})_{6}{ }^{3 / 4-}$ in $0.1 \mathrm{M}$ aqueous $\mathrm{KCl}$ as the redox probe, obtained at cysteamine and glutaraldehyde-cysteamine. When the electrode surface is modified, the electron transfer kinetics of $\mathrm{Fe}(\mathrm{CN})_{6}^{3-/ 4-}$ is perturbed. As shown in Fig. 2, the stepwise assembly of cysteamine and glutaraldehyde molecule on quartz crystal gold electrode is accompanied by a decrease in the amperometric response of the electrode of the redox probe. This is consistent with the enhanced electron transfer barriers introduced upon the assembly of these layers [25, 26] and indicates that cysteamine SAMs was successfully assembled. The differences in the length of carbon chains of monomers may lead to distinctions in the packing of the SAM formed by these thiols. For the alkanethiols with 


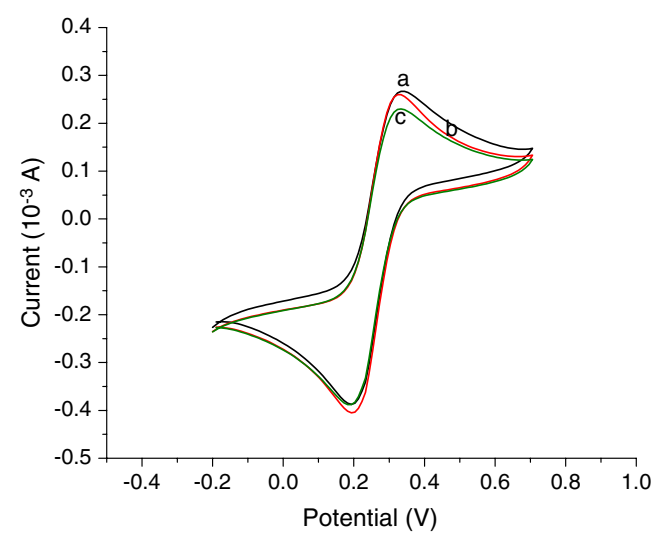

Fig. 2 Cyclic voltammogram in the aqueous solution with $1 \mathrm{mM}$ $\mathrm{K}_{3} \mathrm{Fe}(\mathrm{CN})_{6}^{3 / 4-}$ in $0.1 \mathrm{M} \mathrm{KCl}$ at $100 \mathrm{mV} \mathrm{s}^{-1}$ of stepwise of the SAM preparation on the quartz crystal electrode: (a) bare electrode, (b) cysteamine, and (c) glutaraldehyde

shorter chain length (cysteamine), the decrease on the redox peaks is lower than obtained with long chain length [27].

Quantitative analysis of coverage can be calculated by cyclic voltammetric investigations. The surface coverage $(\theta)$ was calculated by Eq. 2 , where $Q_{\text {Bare }}$ is the charge of the bare quartz crystal electrode and $Q_{\mathrm{SAM}}$ is the charge of the modified electrode by cysteamine, calculated from the area under the peak redox from cyclic voltammogram (Fig.2). The surface coverage of cysteamine by monolayer was $0.0367 \%$ according to Eq. 2, assuming that the SAM formation process was described by simple Langmuir adsorption kinetics. The presence of glutaraldehyde in SAM provided a congenial microenvironment similar to a protein in a native system, thus retaining their bioactivity and allowing the protein ( $r$ Lci2B-NH6) more freedom in orientation. This is due to histidine tail by amine groups and is thus facilitated by a closer approach of the protein to the sensing surface.

$\theta=1-\left(Q_{\text {Bare }} / Q_{\text {SAM }}\right)$

\section{The immunosensor response}

In the liquid medium, there are some parameters that influence the oscillation frequency of crystal. In addition to the thickness, density and the shear modulus of the quartz wafer which change the resonant frequency of the crystal; physical parameters of the adjacent medium such as density or viscosity of the liquid can cause fluctuations during the measurements [28]. These last parameters, resulting from the different liquid media used, such as sample and carrier buffer, need to be corrected to avoid fluctuations on the analytical response. For this reason, in all measurements, the electrical frequencies were obtained in PBS to correct the baseline. The calibration curve was obtained by successive incubations with same canine serum of 1:400 diluted in PBS $(200 \mu \mathrm{L})$. The samples containing antibodies against $L$. chagasi were incubated for $15 \mathrm{~min}$. Prior to frequency measurements, the crystal was previously PBS washed. The immunosensor response was proportional to number of incubation and resulted in a good linearity $(r=0.9872, p<0.01, n=13)$ with a low relative error $(1.89 \%)$ (Fig. 3a). The selectivity of the immunosensor was tested by its incubating with the control (negative serum) and the response was practically constant for different incubations (Fig. 3b).

The levels of antibodies in serum samples were correlated between the proposed immunosensor and ELISA test as standard. It was verified a high correlation occurred between two methods ( $r=0.990, n=7, p<<0.05$ ). Through analysis of paired $t$ test, it was observed that there was no statistically significant difference between the two methods (ELISA and immunosensor; $p=0.3415$ ). Moreover, the serum samples with low values of optical density in ELISA also showed low and almost unchanging values of frequency delta with the immunosensor method (Fig. 3c).

The specificity of an immunosensor is evaluated in relation to the amount of nonspecific bindings that occur on the electrode surface. One way of studying, it is by diluting a serum sample in order to reduce nonspecific bindings until it be possible to discriminate a positive sample. The serum samples were diluted at 1:400, 1:800, 1:1,600, and $1: 3,200$. It was possible to discriminate positive serum of up to 1:1,600 (Fig. 4a). An analysis of the squared correlation coefficients obtained from the calibration curves with different dilution ratio of the canine serum was performed. It was observed a maximal dilution ratio at 1:600, which was able to discriminate a positive canine $L$. chagasi serum. When the canine serum 1:3,200 diluted was tested, the frequencies measured were not linear dependent (Fig. 4b). Regarding sensitivity, this immunosensor showed to be capable to detect a lower antigen concentration $(3 \mu \mathrm{g} / \mathrm{mL})$ than the ELISA method [29, 30].

The parasitological diagnosis in samples of bone marrow aspiration biopsy, lymph nodes or spleen obtained by fine needle aspirative biopsy, still considered the gold standard methods for confirming canine leishmaniasis. In laboratory, such samples can be directly examined by microscope, utilized in PCR tests, seeded in culture medium for Leishmania or even subjected to immunochemical techniques with monoclonal antibodies to find and/or quantify the parasites. However, they present a greatly variable sensitivity, which depends not only on the expertise of the analyser [6], but also on the distribution of parasites in those dog tissues. For instance, a given dog can be infected, but having none parasites in a possibly sampled bone marrow. For this reason, nowadays, the combination of two serological methods (ELISA and IFAT) is frequently used for a certain diagnosis [2], despite time consuming and 


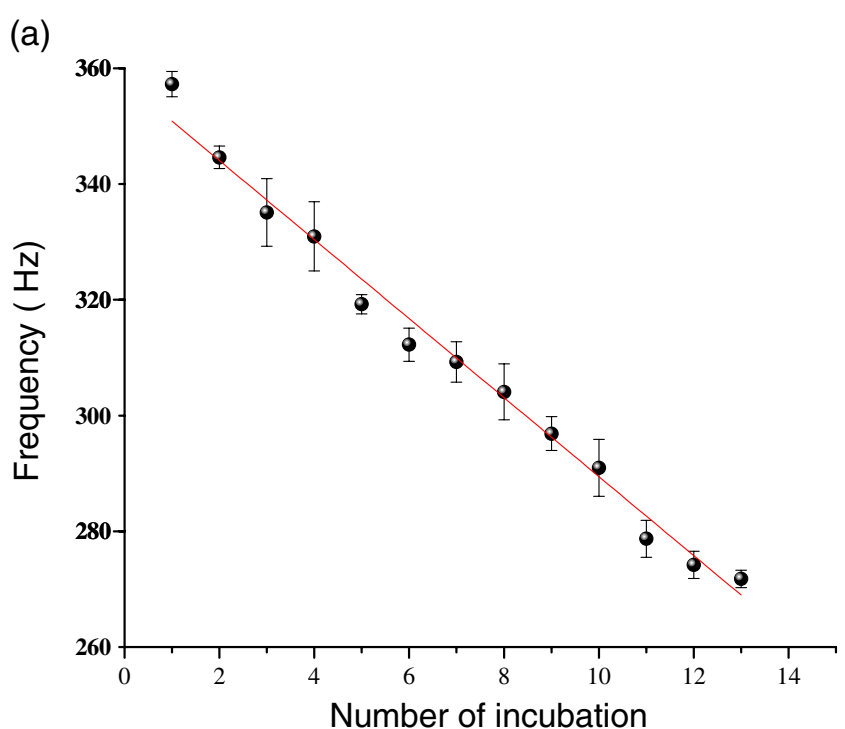

(b)

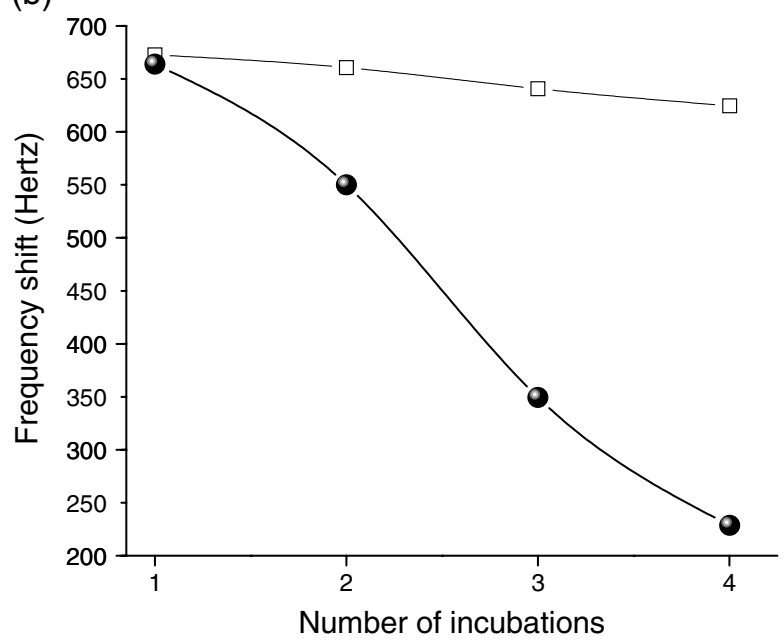

(c)

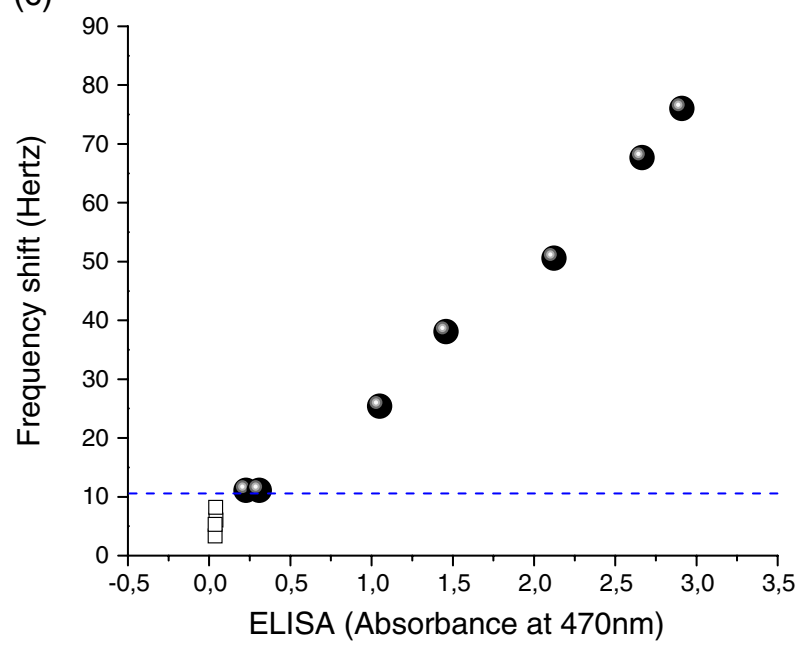

expensive. In the present study, the immunosensor method showed high sensitivity and specificity with regards to the
Fig. 3 Calibration curve of the biosensor for L. chagasi for incubation of canine serum positive for L. chagasi in dilution of 1:400 (a). Comparison of frequency response to incubation of positive $\rightarrow-$ and negative - : - serum samples (b). Correlation between the ELISA (absorbance at $470 \mathrm{~nm}$ ) and the shift frequency $(\mathrm{Hz})$ : the cutoff was established at $10 \mathrm{~Hz}$ of the shift frequency

parasitological biopsy tests, by using the cutoff at $10.5 \%$ $\mathrm{Hz}$, as previously established (Fig. 3c). Thus, this assay may be a promising tool to be used in clinical diagnosis, since it is practical, reliable, and fast

\section{Reuse of the immunosensor}

The reuse of the immunosensor is an important challenge in order to obtain a low-cost analytical method. One of the most important aspects is related to the preservation of the

(a)

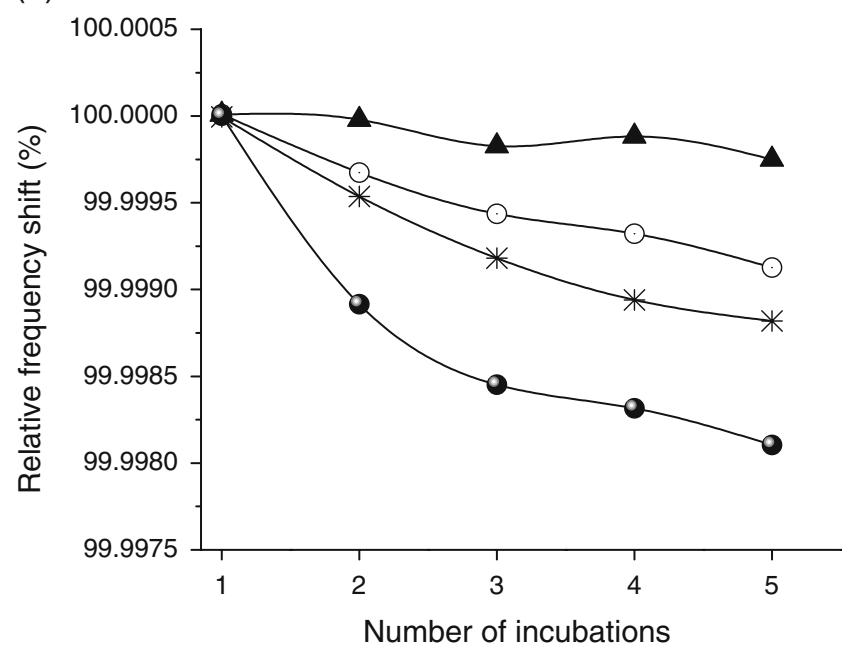

(b)

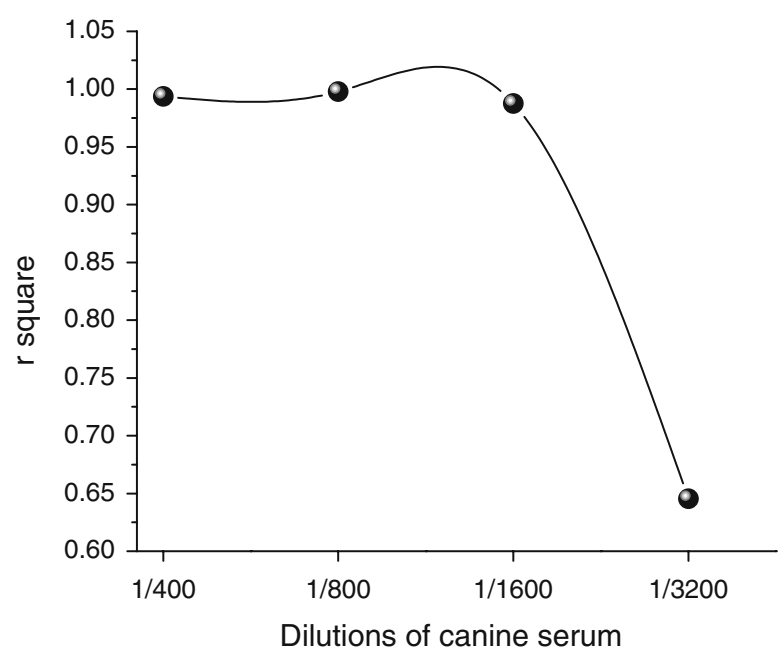

Fig. 4 (a) Response of piezoelectric immunosensor to incubations with positive serum dilutions at $(\boldsymbol{\Delta})$ 1:3,200, (O) 1:1,600, (*) 1: 800, and (1:400. (b) Plot of $r$ square as a function of serum dilutions (b) 
(a)

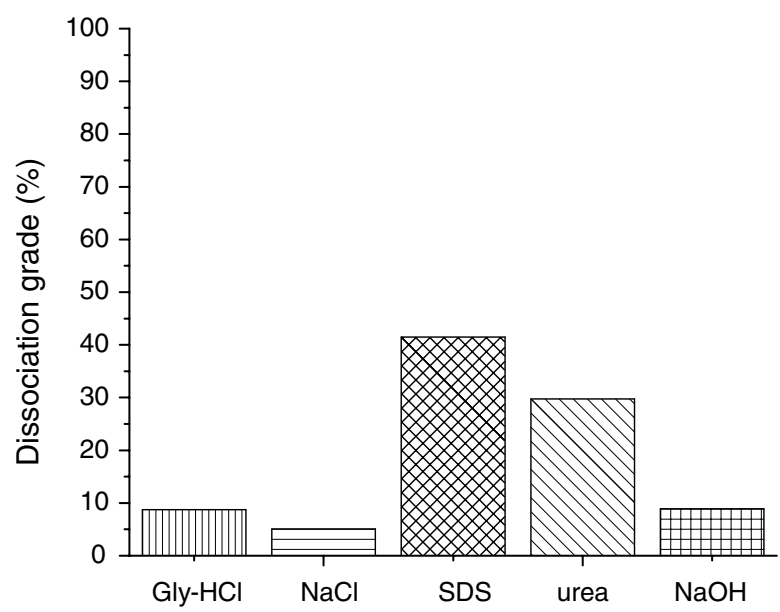

(b)

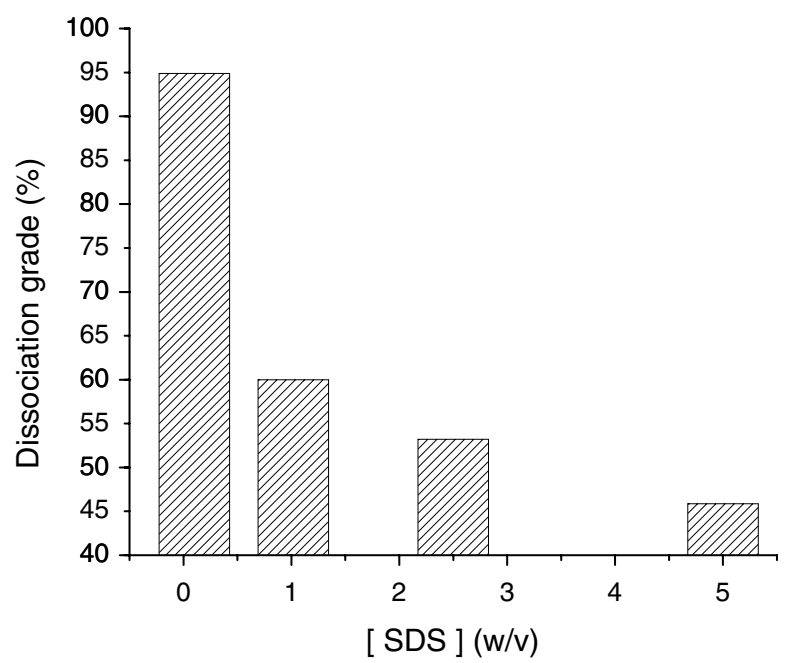

(c)

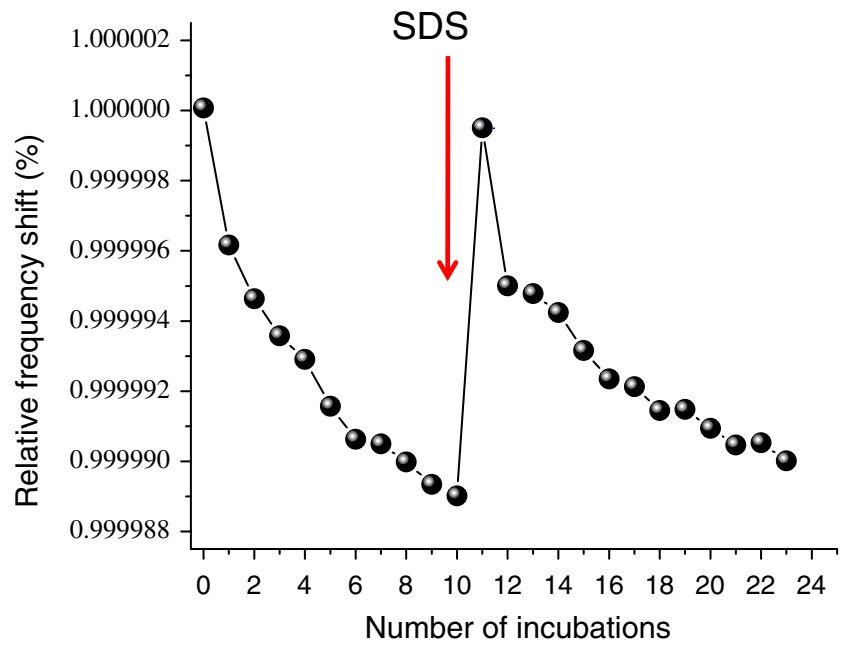

immunoreactivity of the immobilized antigen on the surface of the quartz crystals electrode. The reuse is possible by dissociation of antigen-antibody complex using acidic or
Fig. 5 Comparative study of the dissociation grade (in \%) of antigenantibody using different agents (a). Dissociation grade (in \%) at different concentration of SDS solution $(0.1 \%, 1 \%, 2.5 \%$, and $5 \%)$ (b), and typical assay of dissociation of the immunocomplex after $0.1 \%$ of SDS (c)

basic solution, however high concentrations of these solutions can lead to an inactivation of the immobilized molecule [31]. In this work, different dissociation agents were tested to remove the bound antibodies: $25 \mathrm{mM}$ glycine- $\mathrm{HCl}(\mathrm{pH} 2.3), 0.5 \mathrm{M} \mathrm{NaCl}(\mathrm{pH} 7.0), 0.1 \%$ SDS (pH 6.5), $2 \mathrm{M}$ urea (pH 7.2), and $0.2 \mathrm{M} \mathrm{NaOH}(\mathrm{pH} \mathrm{8.0)}$. The frequency shift observed on addition of these solutions was used to measure the dissociation grade of the antigenantibody complexes. There were no significant differences in the dissociation grade when $\mathrm{NaCl}$, glycine- $\mathrm{HCl}$, and $\mathrm{NaOH}$ solutions were added to the quartz crystal electrode for $1 \mathrm{~min}$. In this case, it was observed a dissociating grade

(a)

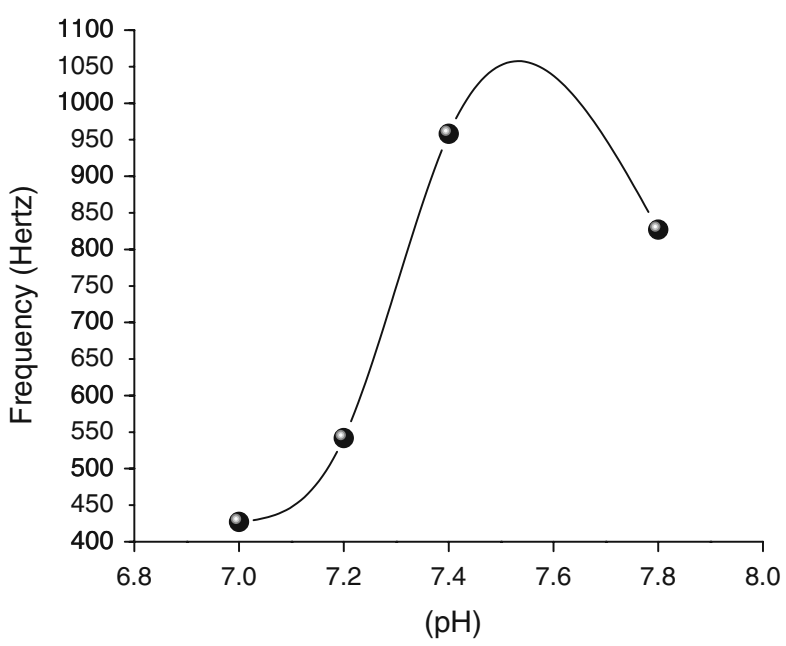

(b)

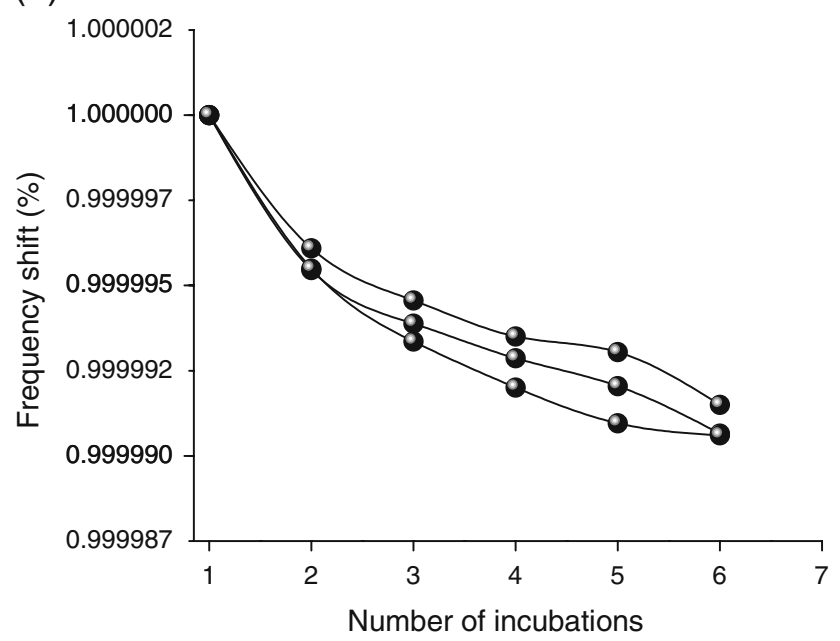

Fig. 6 Immunosensor response as function of $\mathrm{pH}$ (a) and reproducibility of the immunosensor from three different electrodes response (b) 
of $5.06 \%, 8.75 \%$, and $8.90 \%$, respectively. An intermediate dissociation grade using $2 \mathrm{M}$ urea solution was achieved (29.77\%). The highest dissociation grade was obtained $(41.49 \%)$ by incubation with $0.1 \%$ SDS solution (Fig. 5a).

When choosing the antigen-antibody, dissociating agent, other important parameters should be considered such as the preservation grade of the immunoreactivity of immobilized biomolecules, because aggressive solvents can significantly alter their behavior and overall performance [3234]. In this study, the use of SDS provided a high degree of dissociation with maintenance of reactivity, probably due to the SDS $\mathrm{pH}$ being near the neutral $\mathrm{pH}$ and physiological. In the immunosensor area, surfactants and other chaotropes are often used to disrupt a biorecognition element/analyte binding event in order to reset high affinity bioprobes for reuse $[35,36]$. Thus, the SDS was selected as dissociation agent to the following assays.

Additional studies applying the SDS solution for $3 \mathrm{~min}$, showed that a concentration of $0.1 \%$ SDS was optimally considered for dissociation of antigen-antibody interaction (Fig. 5b). This concentration produced $95 \%$ of dissociation. Moreover, in this concentration the antigen activity was preserved, after dissociation, and the baseline was restored permitting new incubations of canine serum (Fig. 5c).

$\mathrm{pH}$ dependence and reproducibility on the immunosensor response

The $\mathrm{pH}$ of the canine serum was studied with regards to the response to the immunosensors, by incubating $200 \mathrm{~mL}$ of canine serum at 1:400 dilution on the quartz crystal electrode in $10 \mathrm{mM}$ PBS. The optimal frequency responses were established $\mathrm{pH} \mathrm{7.4,} \mathrm{that} \mathrm{is} \mathrm{also} \mathrm{similar} \mathrm{to} \mathrm{the}$ physiological $\mathrm{pH}$ of the canine serum, then this $\mathrm{pH}$ was chosen for all remaining studies (Fig. 6a).

It was calculated that the coefficient of variation between assays obtained from three different calibration curves (three electrodes) was equal to $r^{2}=0.983(n=7)$. These data were processed in ORIGIN software version 8.0, (Microsoft, USA). The value of coefficient of variation between assays was of $7 \%$. The analysis of frequencies shift by successive incubations of canine serum at 1:400 dilution on the surface of the three crystals with immobilized antigen rLci2B-NH6 showed that the normalized frequency responses between the crystals did not show statistically significant difference. The analysis of variation within assay showed a good correlation coefficient $(r=0.981)$ and a high reproducibility, with a coefficient of variation within test of $6 \%$ (Fig. 6b).

This reliable immunosensor can be applied to monitor the canine visceral leishmaniasis in serum samples of dogs which can consequently lead to greater transmission control of the parasite to man.

\section{Conclusions}

The QCM immunosensor based on the rLci3B recombinant antigen was successfully applied to the analysis of antibodies against $L$. chagasi in canine serum up to $1: 3,200$ diluted. This immunosensor is a direct method of anti- $L$. chagasi antibodies measurement, without involving marker, making the test faster and simpler than conventional methods. This immunosensor can be portable and is practical for screening testing use in endemic areas of the world and it can be applied to monitor canine visceral leishmaniasis, using serum samples of dogs from endemic areas which can consequently help to improve the transmission control of American visceral leishmaniasis.

Acknowledgment This work was supported by the National Council of Scientific and Technological Development (CNPq) agency of Brazil.

\section{References}

1. Bravo L, Frank LA, Brenneman KA (1993) Canine leishmaniasis in the United States. Comp Cont Educ Prct 15:699-705

2. Moreno J, Alvar J (2002) Canine leishmaniasis: epidemiological risk and the experimental model. Trends Parasitol 18:399-405

3. Ferrer L, Leishmaniasis: update in diagnosis and therapy (1997) In: Proceedings of the Fourteenth Annual Congress of the European Society of Veterinary Dermatology, Pisa. pp 33-36

4. Lachaud L, Chabbert E, Dubessay P, Reynes J, Lamothe J, Bastien P (2001) Comparison of various sample preparation methods for PCR diagnosis of visceral leishmaniasis using peripheral blood. J Clin Microbiol 39:613-617

5. Bourdoiseau G, Bonnefont C, Hoareau E, Boehringer C, Stolle T, Chabanne L (1997) Specific IgG1 and IgG2 antibody and lymphocyte subset levels in naturally Leishmania infantuminfected treated and untreated dogs. Vet Immunol Immunopathol 59:21-30

6. Mancianti F, Pedonese F, Poli A (1996) Evaluation of dot enzymelinked immunosorbent assay (dot-ELISA) for the serodiagnosis of canine leishmaniasis as compared with indirect immunofluorescent assay. Vet Parasitol 65:1-9

7. Figueiredo B, Madeira MF, Nascimento LD, Abrantes TR, MoutaConfort E, Passos SRL, Schubach TMP (2010) Canine visceral leishmaniasis: study of methods for the detection of igg in serum and eluate samples. Rev Inst Med Trop Sao Paulo 52(4):193-196

8. Rodgers MR, Popper SJ, Wirth DF (1990) Amplification of kinetoplast DNA as a tool in the detection and diagnosis of Leishmania. Exp Parasitol 71:267-275

9. Wang H, Lei C, Li J, Wu Z, Shen G, Yu R (2004) A piezoelectric immunoagglutination assay for Toxoplasma gondii antibodies using gold nanoparticles. Biosens Bioelectron 19:701-709

10. Lu F, Lee HP, Lim SP (2004) Quartz crystal microbalance with rigid mass partially attached on electrode surfaces. Sens Actuators A- Phys A 112:203-210

11. Medyantseva EP, Khaldeeva EV, Budniko GK (2001) Immunosensors in biology and medicine: analytical capabilities, problems, and prospects. J Anal Chem 56:886-890

12. Ferreira GNM, da-Silva A-C, Tomé B (2009) Acoustic wave immunosensors: physical models and biological applications of quartz crystal microbalance. Trends Biotechnol 27:689-697 
13. Pan W, Durning CJ, Turro NJ (1996) Kinetics of alkanethiol adsorption on gold. Langmuir 12:4469-4473

14. Kubar J, Fragaki K (2005) Recombinant DNA-derived Leishmania proteins: from the laboratory to the field. Lancet Infect Dis 2:107114

15. Silvia F, Sally P, David AR, Kim ES (2000) Self-assemblead monolayeres: a versatile tool for the formulation of the biosurfaces. TrAC-Trends Anal Chem 9:530-540

16. Jyoung JY, Hong S, Lee W, Choi JW (2006) Immunosensor for the detection of Vibrio cholerae using surface plasmon resonance. Biosens Bioelectron 21:2315-2319

17. Huangxian Ju, Zhao H (2005) Eletrochemical biosensors for DNA analysis. Front Biosci 10:37-46

18. Dutra RF, Mendes RK, Silva VL, Kubota LT (2007) Surface plasmon resonance immunosensor for human cardiac troponin $\mathrm{T}$ based on self-assembled monolayer. J Pharm Biomed Anal 43:1744-1750

19. Badaro R, Benson D, Eulalio MC, Friere M, Cunha S, Netto EM, Pedral-Sampiao D, Madureira C, Burns JM, Hoghton RL, David JR, Reed SG (1996) rK39: a cloned antigen of Leishmania chagasi that predicts active visceral leishmaniasis (VL). J Infect Dis 173:758-761

20. Kumar R, Pai K, Pathak K, Sundar S (2001) Enzyme-linked immunosorbent assay for recombinant $\mathrm{K} 39$ antigen in diagnosis and prognosis of Indian visceral leishmaniasis. Clin Diagn Lab Immunol 8:1220-1224

21. Chaki NK, Vijayamohanan K (2002) Self-assembled monolayers as a tunable platform for biosensor applications. Biosens Bioelectron 17:1-12

22. Campuzano S, Pedrero M, Montemayor C, Fatás E, Pingarrón JM (2006) Characterization of alkanethiol-self-assembled monolayersmodified gold electrodes by electrochemical impedance spectroscopy. J Electroanal Chem 586:112-121

23. Schlereth DD, Schmidt HL (1995) Spectroelectrochemical detection of phenothiazine and phenoxazine derivatives covalently bound to self-assembled cystamine monolayers. J Electroanal Chem 380:117-125

24. Katz E, Schlereth DD, Schmidt HL (1994) Electrochemical study of pyrroloquinoline quinone covalently immobilized as monolayer onto a cystamine modified gold electrode. J Electroanal Chem 367:59-70
25. Xiao-Ya Hu, Yi X, Hong-Yuan C (1999) Adsorption characteristics of $\mathrm{Fe}(\mathrm{CN})_{6}{ }^{3-/ 4-}$ on $\mathrm{Au}$ colloids as monolayer films on cysteamine-modified gold electrode. J Electroanal Chem 466:26-30

26. Cai H, Xu C, He P, Fang Y (2001) Colloid Au-enhanced DNA immobilization for the electrochemical detection of sequence specific DNA. J Electroanal Chem 510:78-85

27. Campuzano S, Galvez R, Pedrero M, de Villena FJM, Pingarron JM (2002) Preparation, characterization and application of alkanethiol self-assembled monolayers modified with tetrathiafulvalene and glucose oxidase at a gold disk electrode. J Electroanal Chem 526:92-100

28. Auge J, Hauptmann P, Hartmann J, Rösler S, Lucklum R (1995) New design for QCM sensors in liquids. Sens Actuators B-Chem 24:43-48

29. Taran M, Mohebali M, Modaresi MH, Mamishi S, Mojarad M, Mahmoudi M (2007) Preparation of a K39sub recombinant antigen for the detection of Leishmania infantum antibodies in human: a comparative study with an immunochromatographic test and direct agglutination. Iranian J Parasitol 2:25-33

30. Mohebali M, Taran M, Zarei Z (2004) Rapid detection of Leishmania infantum infection in dogs: comparative study using an immunochromatographic dipstick rk39 test and direct agglutination. Vet Parasitol 121:239-245

31. Katakura Y, Miyazaki T, Wada H, Omasa T, Kishimoto M, Goto Y, Suga K-I (2000) Control of antibody-antigen ineraction using anion-induced conformational change in anigen peptide. Protein Eng 13:719-724

32. Gekko K, Morikawa T (1981) Thermodynamics of polyol-induced thermal stabilization of chymotrypsinogen. J Biochem 90:51-60

33. Otting G, Liepinsh E, Wuthrich K (1991) Protein hydration in aqueous solution. Science 254:974-980

34. Takeda K, Harada K, Yamaguchi K, Moriyama Y (1994) Conformational changes of bovine serum albumin in an aqueous solution of sodium bis (2-ethyl-hexyl) Sulfosuccinate and in the reverse micelle of the same surfactant. J Colloid Interface Sci 164:382-386

35. Janata J (1989) In: Wolfbeis OS (ed) Fiber optic chemical sensors and biosensors. CRC Press, Boca Raton

36. Betts TA, Catena GC, Huang J, Litwiler KS, Zhang J, Zagrobelny JA, Bright FV (1991) Fiber-optic-based immunosensors for haptens. Anal Chim Acta 246:55-63 
Copyright of Analytical \& Bioanalytical Chemistry is the property of Springer Science \& Business Media B.V. and its content may not be copied or emailed to multiple sites or posted to a listserv without the copyright holder's express written permission. However, users may print, download, or email articles for individual use. 